\title{
Kommunikationsfreiheit im digitalen Zeitalter
}

\author{
Zwölf Thesen. Von Horst Pöttker
}

\begin{abstract}
Die Digitalisierung hat eine starke Ausweitung von Kommunikationsmöglichkeiten mit sich gebracht. Dabei wird die zuvor medientechnisch verankerte Trennung von Massenkommunikation (Öffentlichkeit) und Individualkommunikation (Privatheit) sukzessive aufgehoben. So ist es möglich, dass herkömmliche, rechtlich (Art. 5, Abs. 2 GG) und berufsethisch (Pressekodex) geltende Schranken der Pressefreiheit in sozialen Netzwerken unterlaufen und damit wirkungslos werden. Um auch im digitalen Bereich (wieder) notwendige Schranken der Kommunikationsfreiheit zur Geltung zu bringen, ohne die hier gegebenen Chancen für das Herstellen von Öffentlichkeit zu verbauen, muss entweder journalistische Berufsethik zum Bestandteil der allgemeinen Moral oder die staatliche Regulierung publizistischer Medien auf Netzbetreiber erweitert werden. Der erste Weg ist sehr lang, der zweite mit dem Risiko einer Instrumentalisierung für Machtzwecke belastet.
\end{abstract}

\section{Kommunikation als Menschenrecht}

Bekanntlich ist es unmöglich, nicht zu kommunizieren. Kommunikation ist eine unverzichtbare Grundlage der menschlichen Lebensweise. Als Mängelwesen, die hilflos auf die Welt kommen, sind Menschen auf Kommunikation angewiesen, weil der Austausch von Zeichen und ihren Bedeutungen - Wahrnehmungen, Erfahrungen, Wissen, Ideen, Emotionen - notwendig ist, damit soziale Gebilde entstehen können, in deren Schutz Sozialisation geleistet und Kultur hervorgebracht werden kann. Fasst man in der Tradition der europäischen Aufklärung die revolutionäre Parole „liberté, egalité, fraternité“ als Formel auf, die die Menschenrechte zusammenfasst, ist „fraternité“ („Brü-
Prof. em. Dr. Horst

Pöttker lehrte bis 2013 Journalistik an der TU Dortmund und ist seit 2015 Seniorprofessor an der Universität Hamburg. Er war 2004 (Mit-)Gründer des

„Vereins zur Förderung der publizistischen Selbstkontrolle"(FPS) und von 2002 bis 2014 Geschäftsführer der "Initiative Nachrichtenaufklärung"(INA). 
derlichkeit", heute besser Geschwisterlichkeit oder noch besser Zwischenmenschlichkeit) der Begriff, der in Anbetracht der konstitutiven Bedeutung der Kommunikation für die menschliche Lebensweise ein allen Menschen zustehendes Grundrecht auf Kommunikation zum Ausdruck bringt.

\section{Medienentwicklung vervielfältigt Kommunikationsmöglichkeiten}

Ursprünglich haben Menschen für ihre Kommunikation nur elementare Medien wie Luft und Licht genutzt. Der Austausch von sprachlichen Zeichen war durch die Reichweite des Schalls, der Sicht und der Erinnerung begrenzt und spielte sich zwischen Anwesenden ab. Entsprechend klein waren die sozialen Gebilde, die solche Kommunikation hervorbringen und in deren Rahmen sie sich vollziehen konnte: Familien, Clans, Horden, Dörfer. Mit der Entdeckung und organisatorischen Entfaltung technischer Medien wie Schrift, Druck, Telegraph, Telefon, Film, Schallplatte, Radio, Fernsehen oder Computer sowie deren (digitaler) Vernetzung haben sich Reichweite, Menge und Art von Bedeutungsübertragungen zwischen Menschen erweitert und differenziert. Entsprechend sind Umfang und Vielfalt der durch Kommunikation konstituierten sozialen Gebilde gewachsen.

\section{Trennung von privater und öffentlicher Kommunikation}

Mit der Erweiterung der Kommunikationsmöglichkeiten im Zuge der Medienentwicklung vollzog sich am Beginn der Moderne eine Trennung der unmittelbaren Kommunikation in Kleingruppen von der technisch vermittelten, an viele Empfänger gerichteten Massenkommunikation im Modus „one to many". Da letztere im Prinzip allen Rezipienten offen steht, hat sich dafür der Begriff der „öffentlichen“ Kommunikation eingebürgert, an der wegen ihrer Bedeutung für die ganze Gesellschaft auch politische Funktionen haften. Das Menschenrecht auf Kommunikation umfasst in modernen Großgesellschaften das Recht auf optimale Öffentlichkeit, da ohne eine Sphäre allgemein zugänglicher Information wegen der Komplexität solcher von zahlreichen Kommunikationsbarrieren durchzogenen Gesellschaften allein aufgrund unmittelbarer Erfahrung und direkter interpersonaler Kommunikation weder eine individuelle Lebensgestaltung auf der Höhe der zur Verfügung stehenden Möglichkeiten noch eine Partizipation an sozialen 
Selbstregulierungsmechanismen wie Wahlen oder Märkten gelingen kann.

Für die unmittelbare interpersonale Kommunikation im Modus „between one and one“ oder allenfalls „one and few“, die nur für Mitglieder der jeweiligen Kleingruppe zugänglich ist, bietet sich dagegen der Begriff der privaten oder - die Abgeschlossenheit stärker betonend - der intimen Kommunikation an. Die Trennung der beiden Kommunikationssphären vollzog sich infolge der verwendbaren und tatsächlich verwendeten Medientechnik nahezu von selbst und musste daher kaum durch sozio-kulturelle, beispielsweise rechtliche oder administrative Vorkehrungen hergestellt werden, wurde allenfalls durch sie flankiert.

\section{Freiheit als Merkmal privater Kommunikation}

„Die Gedanken sind frei!“, heißt es in einem Lied, „Wer kann sie erraten?" Da niemand weiß, was nur im Kopf vorgeht, ist Denken, solange es nicht kommuniziert wird, vollkommen frei. Mit der Kommunikation und dem Umfang des Personenkreises, dem sie offen steht, sinkt der Grad der Freiheit. Während öffentliche, allgemein zugängliche Kommunikation zum Schutz von Menschenwürde und Privatsphäre Beschränkungen unterliegt, können unrichtige Behauptungen, Beleidigungen („Schmähkritik“) oder Hassreden („hate speeches“) je unbedenklicher geäußert werden, desto privater und intimer die Kommunikation ist. An Stammtischen werden seit jeher unzutreffende Vorurteile geäußert, wird beispielsweise derb über Nachbarn, Politiker oder Migranten Falsches und Rassistisches geäußert - in der Öffentlichkeit kann das strafbar sein oder Schadenersatz nach sich ziehen.

\section{Freiheit als Problem öffentlicher Kommunikation}

„Jeder hat das Recht, seine Meinung in Wort, Schrift und Bild frei zu äußern und zu verbreiten und sich aus allgemein zugänglichen Quellen ungehindert zu unterrichten. Die Pressefreiheit und die Freiheit der Berichterstattung durch Rundfunk und Film werden gewährleistet. Eine Zensur findet nicht statt" (Art. 5, Abs. 1 GG).

Das deutsche Grundgesetz von 1949 und die Allgemeine Erklärung der Menschenrechte von 1948 beziehen die 
Garantie der Kommunikationsfreiheit nicht nur, aber im Wesentlichen auf öffentliche Kommunikation durch Massenmedien („Pressefreiheit“), weil bei privater oder intimer Kommunikation Freiheit ohnehin in höherem Maße gegeben ist und deshalb nicht ausdrücklich garantiert zu werden braucht. Freiheit öffentlicher Kommunikation dagegen bedarf des Schutzes durch explizite rechtliche und soziale Normen, weil sie stärker politischen, ökonomischen und kulturellen Gefährdungen ausgesetzt ist.

\section{Freiheitsgrenzen als Wesensmerkmal öffentlicher Kommunikation}

„Diese Rechte finden ihre Schranken in den Vorschriften der allgemeinen Gesetze, den gesetzlichen Bestimmungen zum Schutze der Jugend und in dem Recht der persönlichen Ehre" (Art. 5, Abs. 2 GG).

Gleichzeitig damit, dass sie ein Optimum an Freiheit garantieren, setzen grundlegende Regelwerke auch die Grenzen, die bei öffentlicher Kommunikation zum Schutz von Menschenwürde und Privatsphäre notwendig sind. Wissen von und Respekt vor diesen Grenzen sind wesentliche Elemente von Professionalität in den Öffentlichkeitsberufen, die bei Menschen außerhalb dieser Berufe nicht im gleichen Maße zu erwarten sind.

\section{Entgrenzung von privater und öffentlicher Kommunikation}

Die digitale Revolution, ein außerordentlich einschneidender und folgenreicher Umbruch in der Medien- und Kulturentwicklung, hat eine weitere, besonders abrupte und weitreichende Ausdehnung und Vervielfältigung von Kommunikationsmöglichkeiten und -weisen mit sich gebracht. Die technologische Notwendigkeit zur Trennung von privater und öffentlicher Kommunikation ist entfallen. Zwischen die Modi „one to all/ many“ (öffentlich) und „one to one/few“ (privat) schieben sich im globalen Netz der Computer („Internet“) eine Reihe kommunikativer Zwischenformen wie „one to some“, „some to one“, "some to many“, "many to some/one“, "many to many“ usw. In sozialen Netzwerken wird de facto ohne Zugangsbarrieren, also öffentlich kommuniziert, offiziell jedoch im Modus privater Kommunikation ohne inhaltliche Schranken. Vor dem digitalen Umbruch institutionalisierte Mechanismen der ethisch 
und professionell gebotenen Zügelung öffentlicher Kommunikation verlieren ihre Wirksamkeit und werden zu anachronistischen Relikten.

\section{Digitalisierung als Verlust notwendiger Freiheitsgrenzen}

Was bisher aufgrund der begrenzten Reichweite und der darauf beruhenden besonderen Freiheit privater Kommunikation dort an kritischen und, wenn sie allgemein bekannt würden, auch an unliebsamen, verletzenden und inhumanen Inhalten möglich war, dehnt sich infolge der digitalen Weite und Vielfalt an Kommunikationsmöglichkeiten und der verblassenden Grenzen zwischen den Kommunikationssphären nun in den öffentlichen Raum aus, wo ihre Urheber anonym und unerkannt bleiben können. In der digitalen Medienwelt, wie sie gegenwärtig beschaffen ist, können auch unrichtige und beleidigende Inhalte allgemein zugänglich sein, ohne dass den Urhebern eine persönliche Verantwortung zurechenbar ist. Diese neue Schrankenlosigkeit der öffentlichen Kommunikation ist nicht nur ethisch, im Sinne des Menschenrechts auf "persönliche Ehre" problematisch; sie ist auch Faktor einer Verrohung des öffentlichen Diskurses, die den gesellschaftlichen Zusammenhalt gefährdet.

\section{Technologisch erweiterte Kontrolle}

Aus der Digitalisierung folgen aber auch Einschränkungen der Kommunikationsfreiheit. Sie rühren daher, dass staatlichen Organen (Staatsanwaltschaften, Polizei, Geheimdiensten), aber auch Unternehmen und nichtstaatlichen Organisationen nun technologisch effektivere und tiefer greifende Überwachungsinstrumente (digitale Programme, Algorithmen) zur Verfügung stehen, die sich - zumal mit dem rechtfertigenden Hinweis auf den notwendigen Schutz vor illegalen Inhalten - für Kommunikationskontrolle und -lenkung zugunsten politischer und ökonomischer Partikularinteressen nutzen lassen.

\section{Gestärkte Potenziale von Öffentlichkeit}

Gleichzeitig wachsen die Möglichkeiten und damit die Freiheit von Journalist_innen, Whistleblowern und Hackern, aber auch von Regierungen, Missstände festzustellen und bekannt zu machen oder abzustellen. Edward Snowden, WikiLeaks oder die „Panama Papers", aber auch von Finanzministern und Steuerfahndern erworbene Daten-CDs von Banken sind Beispiele. 
Was das Feststellen von Missständen betrifft, spielen digitale Speichermedien betroffener Institutionen eine wichtige Rolle, während für das Bekanntmachen neben herkömmlichen publizistischen Massenmedien soziale Netzwerke an Bedeutung gewinnen.

\section{Staatliche Kontrolle von Netzbetreibern?}

Wenn man nicht auf Habitualisierung notorischer Verletzungen des „Rechts der persönlichen Ehre“ setzen und auf Abstumpfung gegen die Verrohung des öffentlichen Diskurses hoffen will: Was kann gegen die problematischen Auswirkungen der Digitalisierung auf die öffentliche Kommunikation getan werden, ohne die produktiven Potenziale der neuen Kommunikationsfreiheit zu gefährden? In der komplizierten Gemengelage von Chancen und Gefährdungen, Ausweitungen und Einschränkungen, die die Digitalisierung für die Kommunikationsfreiheit mit sich bringt, gilt es, für den Verlust an technologisch bedingter Trennung von privater und öffentlicher Kommunikation rechtliche und ethische Kompensationen zu finden und zu implementieren. Ein Weg sind Einschränkungen von digitalen Netzen durch staatliche Gewalten, die sich technisch vollziehen lassen. Dass sie sich nicht nur - wie in China - für Machtinteressen missbrauchen lassen, sondern auch über (absichtliche?) Missverständnisse zu problematischen Einschränkungen der Informationsfreiheit führen können, hat sich zum Beispiel an der Sperrung des Fotos eines vom Entsetzen gepackten nackten Mädchens im Vietnam-Krieg gezeigt, das vom Netzbetreiber erst nach Protesten wieder zugänglich gemacht wurde. Offenbar bedarf es eines intensiven öffentlichen Diskurses über die notwendigen Schranken der Kommunikationsfreiheit in sozialen Netzwerken, der deren Verantwortliche wirksam erreicht und ihr Handeln gesellschaftlicher Selbstkontrolle unterwirft.

\section{2. „Everybody is a journalist"?}

Eine andere Möglichkeit, der öffentlichen Kommunikation ihre ethisch gebotenen Schranken zurückzugeben, ohne das Grundrecht auf Öffentlichkeit und Informationsfreiheit zu verletzen, ist ein breiter, auch durch Medienpädagogik gestützter kultureller Lernprozess, der den hohen Wert sowohl freier Öffentlichkeit als auch ihrer notwendigen Schranken (Persönlichkeitsrecht, Informationsrichtigkeit) sowie die Sensibilität für deren Unterscheidung zu Bestandteilen der allgemeinen, 
schon in der kindlichen Sozialisation angeeigneten allgemeinen Moral werden lässt: ein langwieriger Prozess, in dessen Verlauf Öffentlichkeitsberufe wie der Journalismus eines ihrer Professionalitätsmerkmale - das nicht einmal alle Journalist_innen realisieren - an die Allgemeinheit abgeben müssten. Alternativen zu diesen beiden steinigen Wegen sind nicht $\mathrm{zu}$ erkennen. 